\title{
Made-to-measure Microbes
}

In 2002, Eckard Wimmer and colleagues, of Stony Brook University in New York, announced to the world that they had assembled a poliovirus from off-the-shelf chemicals, which Wimmer says immediately "caught global attention, high praise, ridicule, and fierce condemnation." Not only did this feat of genetic ingenuity provide "proof of principal," Wimmer stresses, but "the first cell-free viral synthesis was a biological milestone, encouraging large-scale genomic changes." Traditional molecular biology does not allow scientists to make massive alterations in genome architecture; Wimmer emphasizes the usefulness of synthetic genome-building technology for the study of things such as gene function and pathogenicity.

Their synthetic poliovirus-poliovirus type 1 (Mahoney), or sPV1(M) may start off as a chemical, but it quickly comes to life once inside a cell. However, because "watermarks" (genetic markers) were synthesized into sPV1(M), it ended up five orders of magnitude less virulent in mice than its wild prototype, although a high-enough inoculation could still kill. "Unfortunately, this fascinating dual nature of viruses as both nonliving and living entities - that is, chemicals with a life cycle-has largely been ignored,' Wimmer says.

However, Wimmer notes that because their synthesized poliovirus is nearly identical in genotype to the wild-type virus, it is not yet a product of synthetic genomics - which he says, "strives to generate biological systems that don't exist in nature."

Achieving the ideal synthetically programmed microorganism, according to an editorial in Nature Biotechnology (December 2009), requires that we "move away from laboratory-adapted versions of 'natural' organisms-those that bring the genetic and metabolic "baggage' of millions of years of evolution." Although this baggage is essential for wild microbes facing "environmental instability and insult, reproductive fitness, invaders and predators, and the other rigors of survival," it is redundant in the "cushy, constant, cosseted, aseptic, and substrate-rich environs of man-made production systems." The model industrial microbe is an extreme specialist honed to metabolic perfection, and, for the worriers out there, one that would not last a second in the outside world.

Notably, though, synthetically constructed viral genomes that have lost their pathogenic potential can still elicit an immune response in the host; thus, vaccine development is one of the research goals that Wimmer-lab researchers are actively pursuing.

RNA viruses such as polioviruses are synthesized and sequenced with "reverse genetics," which entails converting the RNA genome into doublestranded DNA with the help of a retroviral enzyme known as reverse transcriptase. Synthesizing DNA viruses is quite another thing, and only one- $\Phi X 174$ - has been assembled so far. Hamilton Smith and colleagues in J. Craig Venter's lab worked from a computer-based DNA sequence to assemble the $\Phi X 174$ genome in a test tube, and then activated the resulting DNA into virions in the workhorse bacterium Escherichia coli. "The E. coli cellular machinery read the synthetic genetic DNA and produced the viral proteins, which self-assembled to form the active virus," according to Venter.

But $\Phi$ X174 is also a copy of an existing virus-a bacteriophage, actually-and is not, technically speaking, a product of true synthetic genomics. Nonetheless, "this elegant work confirms the general utility of DNA synthesis for assembling viral genomes first shown with poliovirus," Wimmer stresses.

Thanks to ever-cheaper technology, there are now more than 2361 complete genetic sequences deposited in the Viral Genome Resource, ready to be downloaded and further investigated. Several of these viruses have already been synthetically reconstructed, including the
1918 Spanish influenza virus that killed more than 50 million people worldwide; the simian immunodeficiency virus (SIVcpz), which infects chimpanzees, providing a natural reservoir for the HIV-1 pandemic; the human endogenous retrovirus, a viral fossil embedded in our genomes; and the SARS (sudden acute respiratory syndrome)-like coronavirus. The common goal of this work, according to Wimmer, "is to better understand an organism's properties, particularly its pathogenic armory."

The chemical assembly of viruses was quickly dwarfed in scale by the synthesis of the 582,970-base-pair genome of the bacterium Mycoplasma genitalium. In the 1990s, Venter, Smith, and Clyde Hutchison sequenced its genome and then set about knocking out unwanted genes with the aim of selecting viable cells with the smallest number of housekeeping genes possible. "Easy to say," observes Venter, but "extremely hard and frustrating to carry out." Eventually it was decided that the only way to make a minimal genome would be to chemically synthesize the chromosome, physically vary the gene content, and then put it back into a gutted bacterium; indeed, the synthesis of $\Phi X 174$ was a test run for this endeavor.

"Although not yet 'booted to life, the assembly of such a large DNA molecule bears witness to the vast possibilities DNA synthesis will ultimately offer," Wimmer says. This may soon come. As predicted in the 7 January issue of Nature, "surely, this will be the year when genome pioneer Craig Venter and his team reveal they have booted up a laboratory-made genome inside a living bacterial cell, to create what will be billed as synthetic life." Venter too thinks a synthetically constructed bacterium will happen soon, but reminds the scientific community that he has been saying this for the past two years now.

Marcia Stone (www.mstoneworks.net) is a science writer based in New York City.

doi:10.1525/bio.2010.60.4.16 